Article

\title{
Some Aspects of Sustainable Energy Conversion during Transient Processes in Electric Power Systems Comprising Generator Circuit Breakers
}

\section{Cornelia A. Bulucea ${ }^{1, *}$, Marc A. Rosen ${ }^{2}$, Doru A. Nicola ${ }^{1}$, Nikos E. Mastorakis ${ }^{3}$ and Carmen}

\section{A. Bulucea ${ }^{4}$}

1 University of Craiova, Faculty of Electrical Engineering, Craiova 200440, Romania;

2 Faculty of Engineering and Applied Science, University of Ontario Institute of Technology, Oshawa, ON, L1H 7K4, Canada;

3 Technical University of Sofia, Industrial Engineering Department, Sofia, Bulgaria \& Military Institutions of University Education (ASEI), Hellenic Naval Academy, Piraeus 18539, Greece;

4 University of Medicine and Pharmacy of Craiova, Craiova 200349, Romania; E-Mails: abulucea@gmail.com (C.A.B.); marc.rosen@uoit.ca (M.R.); dorunicola@gmail.com (D.N.); mastorakis4567@gmail.com (N.M.); carmen.bulucea@gmail.com (C.B.)

* Author to whom correspondence should be addressed: E-Mail: abulucea@gmail.com (C.A.B.); Tel.: $+40-724-751-952$

Received: 02 August 2013 / Accepted: 15 September 2013 / Published: 01 November 2013

\begin{abstract}
This paper is a sequel to a study by the authors of electric power systems comprising the generator circuit-breakers (GCBs) at power plant generator terminals. A sustainable assessment of the current interruption requirements of a GCB addresses the main stresses on the generator circuit breaker, revealing that the GCB current interruption requirements are significantly higher than for the distribution network circuit breakers. Hence, generator circuit-breakers are subject to unique demanding conditions caused by specific stresses, namely: high asymmetrical fault currents resulting from high d.c. components of the fault current; greater electrical, thermal and mechanical stresses when interrupting longer arcing time faults; and important dielectric stress after the electric arc extinction caused by the transient recovery voltage (TRV). This paper extends other studies of the authors on the energetic and exergetic transformation chain at the interruption current
\end{abstract}


transient process in an electric power system that comprises the generator circuit-breaker, as well as the transient recovery voltage which appears after the interruption of a short-circuit fed by the synchronous generator or by the main step-up transformer. For achieving the TRV equivalent configuration the authors applied the method of operational symmetrical components (o.s.c.), and utilized the operational impedances of the synchronous generator and of the main transformer, depending on the fault location. Modeling the transient recovery voltage of circuits emphasizes aspects with direct implications on commutation equipment. Thus, the o.s.c. method can be applied at the poles of any breaker, for any eliminated fault type, if the network configuration and elements are known. The TRV, which appears after the interruption of a short-circuit fed by the generator, may be considered like an oscillation, where the oscillation factor and the rising rate (RR) of the TRV are established by the electrical machine parameters: resistance, inductance and capacitance. Consequently, modeling of concentrated equivalent parameters of the synchronous generator at perturbations caused by current interruption transient processes is achieved in this study through an approach based on sustainability concepts. These findings allow for simulations of the transient recovery voltage and comparisons with experimental results.

Keywords: electric arc; exergy; generator circuit-breaker; short-circuit; sustainability; transient recovery voltage

\section{Introduction}

Following the notion that life on Earth demonstrates sustainable energy conversion [1-4], we aim to highlight that an approach to an electrical power system according to the patterns defined within the sustainability framework could provide a synoptic view of electromagnetic interaction phenomena within an electric power network entailing a generator circuit-breaker (GCB) at the terminals of the synchronous generator of an electric power station [5-7].

The electric connection circuits of power stations encompass GCBs at the generator terminals, since the presence of that electric equipment is related to the sustainability of a power plant [4-15]. The location of a GCB, between the generator and the main step-up transformer, influences the operating conditions, and a sustainable assessment of the GCB requirements addresses the main stresses on generator circuit breakers, revealing that the GCBs are significantly more difficult to apply to some operating regimes than classical network circuit-breakers [5-19].

In previous studies [16-19] we have shown the configuration of the key fault current encountered by the generator circuit breaker, namely: generator-fed faults (which can be insulated or grounded threephase and two-phases short-circuits); transformer-fed faults (which can be insulated or grounded threephase and two-phases short-circuits); and generator-fed faults, on the high voltage side of the main step-up transformer (which can be insulated or grounded three-phase and two-phases short-circuits, as well as single-phase short-circuits).

To interrupt these kinds of faults, generator circuit-breakers are subjected to specific stresses: 
- The GCBs must be capable of interrupting not only the high symmetrical fault current, but also the higher asymmetrical faults currents resulting from high d.c. components of the fault current [5-10]. Here arises a sustainability requirement for generator circuit-breakers which are subjected to a unique demanding condition, called delayed current zeros $[5,12,16]$.

- Since circuit breakers interrupt on the current zero crossing, generator circuit-breakers must be able to withstand longer arcing times and greater electrical, thermal and mechanical stresses when interrupting such faults [5-17].

- Just after the short-circuit current interruption by the generator circuit-breaker (when the GCB has been subjected to a very high temperature plasma arc), between its opened contacts arises the transient recovery voltage, which constitutes the most important dielectric stress after the electric arc extinction [12-14, 16-19].

The authors utilized the framework of sustainability and addressed the GCB specific stresses through an exergy analysis of the transformation chain for the interruption current transient process in an electric power system that comprises the generator circuit-breaker [16-17]. The exergy balance of the

interrupted circuit during the arc burning $X_{\text {in }}=X_{\text {out }}+X_{\text {stored }}$ emphasizes that the stored exergy $\mathrm{X}_{\text {stored }}$ is represented by the energy embedded in the magnetic field of the generator windings, corresponding to different states (sub-transient, transient and steady-state short-circuit), and embodied in the arc circuit [1617].

After the electric arc extinction in the GCB, the magnitude and shape of the TRV occurring across the generator circuit-breaker are critical parameters in the recovering gap after the current zero and they are related to further transformation of the exergy stored in the interrupted alternating-current circuit that comprises the synchronous generator and the GCB.

In this paper, for the case of the faults fed by the synchronous generator, we determine the TRV equivalent configuration with operational impedances, taking into account the generator parameters, and applying the operational symmetrical components method.

\section{Modelling of Transient Recovery Voltage (TRV) during Interruption of Short-circuits Fed by the Synchronous Generator}

The dielectric strength within the extinction chamber of the circuit-breaker should increase to a higher value than the TRV; otherwise the electric arc may rekindle [14]. The circuit-breaker capability in interrupting the new arc is strongly affected since the gas-filled space between the two electrodes has an important conductivity and this time the electric arc exergy would be entirely used in a destructive process.

Although the mechanical disconnection of the three phases is performed simultaneously, because of the currents angle phase, the arcs extinction on the phases cannot be simultaneous. It first switches the arc on the phase so its current is passing first through zero. Therefore, the TRV on the three phases are different, even in the same three-phase fault interruption. Moreover, the TRV occurring across the generator circuit breaker depends both on the fault location and on the fault nature [14-22].

In the sustainability framework of this paper, we determine the TRV when the three-phase shortcircuit fed by the synchronous generator is interrupted, using the operational symmetrical components method [16-19]. 
Basically, any non-symmetrical three-phase system of currents or voltages can be decomposed into two symmetrical three-phase systems (of different sequences) and a system of single-phase quantities [20,23-26]. The method application presumes linearity, having been used together with the symbolic representation, in a simplified complex, of the sinusoidal quantities. On basis of this transformation, applied to the phase instantaneous quantities of a three-phase system, we obtain complex quantities (which lose their physical significance), named +,-,0 coordinates or instantaneous symmetrical components. In the paper we follow the same idea of transformation of phase instantaneous quantities at the generator circuit-breaker terminals, in the moments subsequent to its disconnection.

The TRV occurring across the generator circuit breaker is considered only at $t>0$, like a damped oscillation by high frequency $(\mathrm{f}>>50 \mathrm{~Hz})$, starting from zero. Consequently, it is noted that both the TRV and the high frequency currents and voltages achieve the original function conditions.

Applying the operational calculus to the relations determined by the instantaneous symmetrical components method, we obtain the operational symmetrical components (o.s.c.) of the phase voltages and currents [16-19]. Moreover, the o.s.c. of the phase voltages and currents - on the synchronous generator side - are related by operational equations, of the form:

$$
\begin{aligned}
& U_{g+}(p)=Z_{g_{+}}(p) \cdot I_{+}(p) \\
& U_{g_{-}}(p)=Z_{g_{-}}(p) \cdot I_{-}(p) \\
& U_{g_{0}}(p)=Z_{g_{0}}(p) \cdot I_{0}(p)
\end{aligned}
$$

where $Z_{g^{+}}(p), Z_{g_{-}}(p)$ and $Z_{g_{0}}(p)$ denote the phase operational impedances of the synchronous generator in the symmetrical regimes by positive, negative and zero sequences.

Applying the o.s.c. method, the operational image of the TRV occurring across the GB poles after the current interruption can be determined. If the phase current interruption is modelled by the current injection $i_{k}$ - equal but opposite to the eliminated one - the operational image of the TRV occurring across the generator breaker can be expressed in mathematical form:

$$
U_{t r}(p)=-I_{k}(p) \cdot Z_{e}(p)
$$

where $I_{k}(p)$ represents the operational image of the switched current and $Z_{e}(p)$ the operational equivalent impedance across the generator circuit breaker.

We take into consideration the fault location in which the short-circuit currents that must be interrupted by GCB are fed by the synchronous generator. The considered generator has the stator three-phase windings connected by configuration $Y$. Consequently, the GB can be submitted to the insulated or grounded three-phase and two-phases short-circuits. In the paper, for illustration, we analyze the insulated three-phase short-circuit.

The TRV calculation configuration for the moment of the GB first phase opening is represented in Figure 1.

The current interruption has been considered as result of injection on phase $A$ of the current under the restriction:

$$
u_{g B}=u_{g C}
$$


and the TRV occurring at the pole $A-A^{\prime}$ is:

$$
u_{t r}=u_{g A}-u_{g B}
$$

Figure 1. First phase opening at insulated three-phase short-circuit

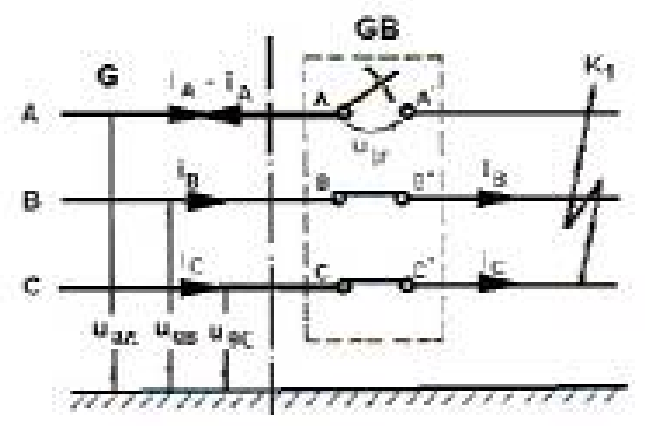

Applying the o.s.c. method, the TRV operational equation takes on the form:

$$
U_{t r}(p)=I_{A}(p) \cdot \frac{3 Z_{g^{+}}(p) \cdot 3 Z_{g_{-}}(p)}{3 Z_{g^{+}}(p)+3 Z_{g_{-}}(p)}
$$

Equation (5) allows the operational representation in Figure 2a.

When $Z_{g^{+}}(p)=Z_{g_{-}}(p)=Z_{g}(p)$, Equation (5) becomes:

$$
U_{t r}(p)=1,5 \cdot I_{A}(p) \cdot Z_{g}(p)
$$

with the operational circuit corresponding to Fig. 2 b.

Figure 2. Operational equivalent circuit: (a) TRV at insulated three-phase short-circuit disconnection, with $Z_{g^{+}}(p) \neq Z_{g_{-}}(p)$; (b) TRV at insulated three-phase short-circuit disconnection, with

$$
Z_{g^{+}}(p)=Z_{g_{-}}(p)=Z_{g}(p)
$$

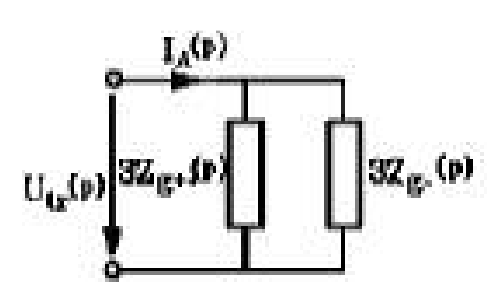

(a)

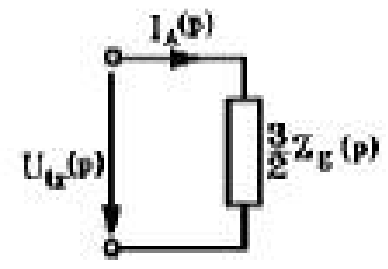

(b)

Applying the method of operational symmetrical components to determine the TRV which appears after the interruption of a short-circuit fed by the generator or the main transformer represents an original contribution of the authors of this study. The method is suitable for application at the poles of any circuit breaker, for any eliminated fault type (fed by the main transformer or by the synchronous generator), if the elements and configuration of the power network are known. Note that, in analytical 
studies, the calculation of the transient recovery voltage at the circuit breakers situated in the proximity of the generators is made on basis of the equivalent configurations with concentrated parameters. The TRV which appears after the interruption of a short-circuit fed by the generator may be considered like an oscillation with the oscillation factor and the rising rate (RR) of TRV established by the electrical machine parameters: resistance, inductance, and capacitance. Consequently, addressing the analysis of synchronous generator behavior during an electromagnetic perturbation (caused by the current interruption) in an electric power system comprising generator circuit-breakers, and the assessment of the values of the concentrated equivalent parameters of the synchronous generators windings follow in this study.

\section{Synchronous Generator Behaviour over the Interruption Processes in an Electric Power System Comprising a GCB}

\subsection{Equations of Synchronous Generator at Electric Perturbation}

In this study we have been adopted the usual hypotheses to define the ideal synchronous machine [20, 23-26], neglecting the iron saturation and hysteresis phenomena and ferromagnetic core losses. The ideal synchronous machine will have the same self and mutual leakage reactances of the stator as the substituted real machine. In terms of the effects that depend on the rotor position, the idealization goes far to considering each stator winding as having a sinusoidal distribution. The positive directions (of voltage and current) are taken into account according to the generator convention.

In Figure 3 there are specified the axes of the phases A, B and C of the ideal synchronous machine, as well as the phase axes $(\alpha, \beta)$ and $(\mathrm{d}, \mathrm{q})$, respectively, of the equivalent synchronous machines; the rotor rotating sense being also specified in Figure 3 . The magnetization directions of currents $i_{A}, i_{B}, i_{C}$, and $i_{\alpha}, i_{\beta}$, respectively, are fixed in the reference frame related to stator, whereas the magnetization directions $i_{d}, i_{q}$ are fixed in the reference frame related to rotor.

Figure 3. Representation of windings and frame systems $(\alpha, \beta)$ and $(\mathrm{d}, \mathrm{q})$ of the synchronous generator

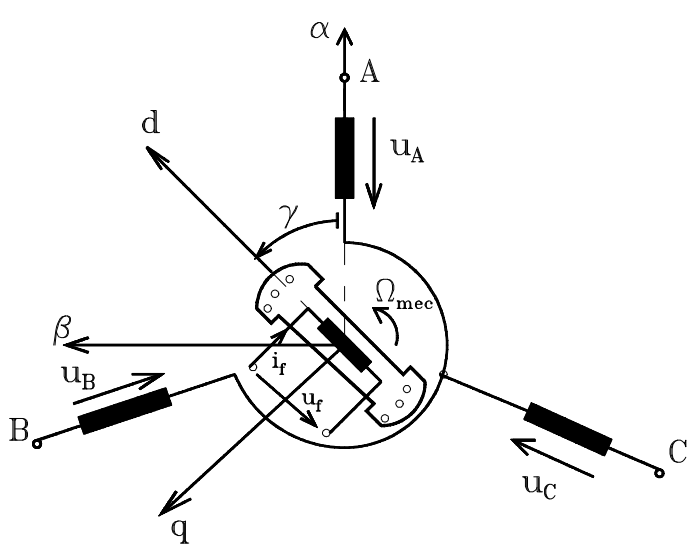

Since through a synchronous generator perturbation a sudden transformation of the operation conditions is understood [23, 26-28], we can assume that the first phase current interruption by the generator circuit-breaker constitutes a synchronous generator perturbation. Because of the extremely small 
perturbation duration, the voltage regulator action is imperceptible. For the same reason it can as well be considered that the rotor position (specified by the angle $\gamma_{0}$, measured between the axis of phase $\mathrm{A}$ and the longitudinal axis d) remains unchanged over the whole perturbation duration.

The sudden variation of the current on one phase (for instance, on phase A) will determine the sudden variations $\Delta \mathrm{i}_{\mathrm{d}}$ and $\Delta \mathrm{i}_{\mathrm{q}}$ of the equivalent machine currents in the frame coordinates $(\mathrm{d}, \mathrm{q})$, as follows:

$$
\Delta_{i_{d}}=\left(2 \Delta_{i_{A}} \cdot \cos \gamma_{0}\right) / 3 ; \Delta_{i_{q}}=-\left(2 \Delta_{i_{A}} \cdot \sin \gamma_{0}\right) / 3
$$

That means the synchronous generator will be submitted to a transient regime. Consequently, at the first moment, assuming the superconducting rotor circuits, the variations of the fluxes linked to the equivalent machine windings in coordinates d,q (caused by the sudden variations of the currents) will be:

$$
\Delta \Psi_{d}=L_{d}^{\prime \prime} \cdot \Delta i_{d} ; \Delta \Psi_{q}=L_{q}^{\prime \prime} \cdot \Delta i_{q}
$$

Considering the recurrence relations between the coordinates $(d, q)$ and $(\alpha, \beta)$ [3-5]:

$$
\begin{aligned}
& \Delta_{i_{d}}=\Delta i_{\alpha} \cdot \cos \gamma_{0}+\Delta i_{\beta} \cdot \sin \gamma_{0} \\
& \Delta_{i_{q}}=-\Delta i_{\alpha} \cdot \sin \gamma_{0}+\Delta i_{\beta} \cdot \cos \gamma_{0}
\end{aligned}
$$

it can be hypothesized that these sudden variations $\Delta \mathrm{i}_{\mathrm{d}}$ and $\Delta \mathrm{i}_{\mathrm{q}}$ would be determined by some correspondent variations $\Delta \mathrm{i}_{\alpha}$ and $\Delta \mathrm{i}_{\beta}$ (unknown, for the time being) of the currents flowing through the stator windings $(\alpha, \beta)$ of the equivalent machine.

Similarly, the fluxes' variations $\Delta \Psi_{\alpha}$ and $\Delta \Psi_{\beta}$ are determined:

$$
\begin{aligned}
& \Delta \psi_{\alpha}=\Delta \psi_{d} \cdot \cos \gamma_{0}-\Delta \psi_{q} \cdot \sin \gamma_{0} \\
& \Delta \psi_{\beta}=\Delta \psi_{d} \cdot \sin \gamma_{0}+\Delta \psi_{q} \cdot \cos \gamma_{0}
\end{aligned}
$$

If relations (9) are substituted in (8), with the further results in (10), we obtain the dependencies among the fluxes' variations $\Delta \Psi_{\alpha}, \Delta \Psi_{\beta}$ and the corresponding currents' variations $\Delta \mathrm{i}_{\alpha}, \Delta \mathrm{i}_{\beta}$ :

$$
\begin{aligned}
& \Delta \psi_{\alpha}=\left(L_{d}{ }^{\prime \prime} \cos ^{2} \gamma_{0}+L_{q}{ }^{\prime \prime} \sin ^{2} \gamma_{0}\right) \Delta i_{\alpha}+\left(L_{d}{ }^{\prime \prime}-L_{q}{ }^{\prime \prime}\right) \sin \gamma_{0} \cos \gamma_{0} \Delta i_{\beta} \\
& \Delta \psi_{\beta}=\left(L_{d}{ }^{\prime \prime}-L_{q}{ }^{\prime \prime}\right) \sin \gamma_{0} \cos \gamma_{0} \Delta_{i_{\alpha}}+\left(L_{d}{ }^{\prime \prime} \sin ^{2} \gamma_{0}+L_{q}{ }^{\prime \prime} \cos ^{2} \gamma_{0}\right) \Delta_{i}
\end{aligned}
$$

With the supplementary notations:

$$
\begin{aligned}
& L_{x}=\left(L_{d}{ }^{\prime \prime}+L_{q}{ }^{\prime \prime}\right) / 2 \\
& L_{y}=\left(L_{d}{ }^{\prime \prime}-L_{q}{ }^{\prime \prime}\right) / 2
\end{aligned}
$$

equation (11) takes the form:

$$
\begin{aligned}
& \Delta \psi_{\alpha}=\left(L_{x}+L_{y} \cos 2 \gamma_{0}\right) \Delta i_{\alpha}+L_{y} \sin 2 \gamma_{0} \Delta i_{\beta} \\
& \Delta \psi_{\beta}=L_{y} \sin 2 \gamma_{0} \Delta i_{\alpha}+\left(L_{x}-L_{y} \cos 2 \gamma_{0}\right) \Delta i_{\beta}
\end{aligned}
$$


The fluxes' variations $\Delta \Psi_{\alpha}$ and $\Delta \Psi_{\beta}$ implicitly determine further voltage variations. These will result from the synchronous generator voltage equations in coordinates $\alpha, \beta$ :

$$
\begin{aligned}
& \Delta u_{\alpha}=-R \cdot \Delta i_{\alpha}-\frac{d}{d t} \Delta \psi_{\alpha} \\
& \Delta_{u_{\beta}}=-R \cdot \Delta i_{\beta}-\frac{d}{d t} \Delta \psi_{\beta}
\end{aligned}
$$

or after substituting the expressions of $\Delta \Psi_{\alpha}$ and $\Delta \Psi_{\beta}$ :

$$
\begin{aligned}
& \Delta u_{\alpha}=-R \Delta_{i_{\alpha}}-\left(L_{x}+L_{y} \cos 2 \gamma_{0}\right) \frac{d}{d t} \Delta i_{i_{\alpha}}-L_{y} \sin 2 \gamma_{0} \frac{d}{d t} \Delta i_{\beta} \\
& \Delta_{u_{\beta}}=-R \Delta_{i_{\beta}}-L_{y} \sin 2 \gamma_{0} \frac{d}{d t} \Delta i_{i_{\alpha}}-\left(L_{x}-L_{y} \cos 2 \gamma_{0}\right) \frac{d}{d t} \Delta i_{\beta}
\end{aligned}
$$

Writing with capitals the operational Laplace images of voltages and currents, the system of equations (15) becomes:

$$
\begin{aligned}
& \Delta U_{\alpha}=-\left\{R+\left(L_{x}+L_{y} \cos 2 \gamma_{0}\right) p\right\} \Delta I_{\alpha}-L_{y} \sin 2 \gamma_{0} p \Delta I_{\beta} \\
& \Delta U_{\beta}=-L_{y} \sin 2 \gamma_{0} p \Delta I_{\alpha}-\left\{R+\left(L_{x}-L_{y} \cos 2 \gamma_{0}\right) p\right\} \Delta I_{\beta}
\end{aligned}
$$

Equations (16) ascertain, in a general case, the link between the current variations and voltage variations at the terminals of the windings $\alpha, \beta$ of the synchronous generator. In the context of some local restrictions, these equations can be simplified, both for short-circuit and load disconnection.

\subsection{First Phase Current Interruption}

The initial conditions corresponding to the three-phase short-circuit at the terminals of the synchronous generator are specified by $\mathrm{u}_{\mathrm{A}}(\mathrm{t})=\mathrm{u}_{\mathrm{B}}(\mathrm{t})=\mathrm{u}_{\mathrm{C}}(\mathrm{t})$. After the current interruption in phase A, the synchronous generator is passing for a short time in a two-phases short-circuit, with $u_{B}(t)=u_{C}(t)$. Calculation of voltage at the terminals of winding $\beta$ :

$$
u_{\beta}=\left\{u_{B}-u_{C}\right\} / \sqrt{3}
$$

emphasizes that on whole perturbation duration $\mathrm{u}_{\beta}=0$, and consequently $\Delta \mathrm{U}_{\beta}=0$ as well.

Therefore, the current variations $\Delta \mathrm{I}_{\alpha}$ and $\Delta \mathrm{I}_{\beta}$ are linked by the relation:

$$
\Delta I_{\beta}=-\frac{L_{y} \cdot \sin 2 \gamma_{0} \cdot p}{R+\left(L_{x}-L_{y} \cdot \cos 2 \gamma_{0}\right) \cdot p} \Delta I_{\alpha}
$$

By substituting further the relation above in expression (16) we obtain: 


$$
\Delta U_{\alpha}=-\frac{R^{2}+2 R \cdot L_{x} \cdot p+\left(L_{x}^{2}-L_{y}^{2}\right) \cdot p^{2}}{R+\left(L_{x}-L_{y} \cdot \cos 2 \gamma_{0}\right) \cdot p} \cdot \Delta I_{\alpha}
$$

Equation (19) constitutes precisely the operational equation of the synchronous generator in the coordinates $\alpha, \beta$ at the current interruption in phase $\mathrm{A}$.

In the case of the load disconnections, after the first phase current interruption, the synchronous generator moves to operate in an unsymmetrical regime.

Noting that the line voltage $\mathrm{u}_{\mathrm{BC}}=\mathrm{u}_{\mathrm{B}}-\mathrm{u}_{\mathrm{C}}$ remains actually unchanged, and also that $\mathrm{u}_{\beta}=\mathrm{u}_{\mathrm{BC}} / \sqrt{3}$, we determine that in this case as well $\Delta \mathrm{U}_{\beta}=0$ over the whole perturbation duration. Consequently, in the framework of load disconnection, the synchronous generator behavior at the first phase current interruption will be depicted as well by equation (19).

Based on the notations (12) for equation (19) we obtain the form:

$$
\Delta U_{\alpha}=-\frac{\left(R+p L_{d}^{\prime \prime}\right)\left(R+p L_{q}^{\prime \prime}\right)}{\left(R+p L_{d}^{\prime \prime}\right) \sin ^{2} \gamma_{0}+\left(R+p L_{q}^{\prime \prime}\right) \cos ^{2} \gamma_{0}} \Delta I_{\alpha}
$$

or:

$$
\Delta U_{\alpha}=-\Delta I_{\alpha} \frac{1}{\frac{R}{\frac{R}{\sin ^{2} \gamma_{0}}+p \frac{L_{q}{ }^{\prime \prime}}{\sin ^{2} \gamma_{0}}+\frac{R}{\cos ^{2} \gamma_{0}}+p \frac{L_{d}{ }^{\prime \prime}}{\cos ^{2} \gamma_{0}}}}
$$

Since the coordinates $\alpha$ of voltages and currents are determined with the following relations:

$$
u_{\alpha}=\frac{2}{3}\left(u_{A}-\frac{u_{B}+u_{C}}{2}\right) ; i_{\alpha}=\frac{2}{3}\left(i_{A}-\frac{i_{B}+i_{C}}{2}\right)
$$

and the considered perturbation solely affects the quantities of phase A, we directly find in operationa that:

$$
\Delta U_{\alpha}=2 / 3 \Delta U_{A} ; \Delta I_{\alpha}=2 / 3 \Delta I_{A}
$$

Further equation (21) takes the form:

$$
\Delta U_{A}=-\Delta I_{A}\left\{\frac{1}{\frac{R}{\sin ^{2} \gamma_{0}}+p \frac{L_{q}^{\prime \prime}}{\sin ^{2} \gamma_{0}}}+\frac{1}{\frac{R}{\cos ^{2} \gamma_{0}}+p \frac{L_{d}{ }^{\prime \prime}}{\cos ^{2} \gamma_{0}}}\right\}^{-1}
$$

Equation (24) allows settling the simplified operational circuit, without considering the capacitance of the synchronous generator at the first phase interruption, as depicted in Figure 4. This is used to assess the equivalent phase circuit structure of the synchronous generator during the current interruption process, with the aim to determine the transient recovery voltage across the generator circuit breaker. 
Figure 4. Simplified operational circuit of synchronous generator at first phase current interruption

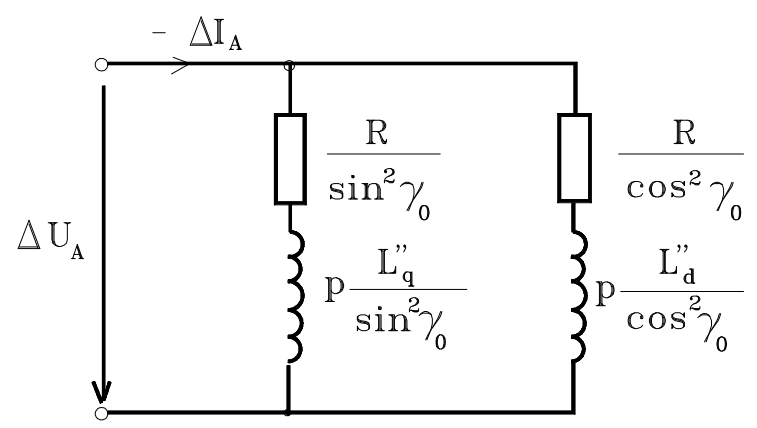

\subsection{Synchronous Generator Operational Circuit at First Phase Current Interruption}

Studies on synchronous generator windings over the interruption processes [27-30] have emphasized the finding that the windings could be seen as long straight conductors. In such situation the capacitance is mainly distributed between each winding and the ferromagnetic stator core, because both the capacitive coupling among the phase windings and the longitudinal capacitance (among winding turns) are very small and can be neglected.

Analyzing the first phase current interruption process when it is considered the equivalent concentrated capacitances $\mathrm{C}_{\mathrm{eg}}$ (see Figure 5), it is ascertained that through the stator winding A:

$$
\Delta I_{A}(p)=I_{c a p}(p)-I_{A}(p)
$$

Figure 5. Equivalent concentrated capacitances at first phase current interruption

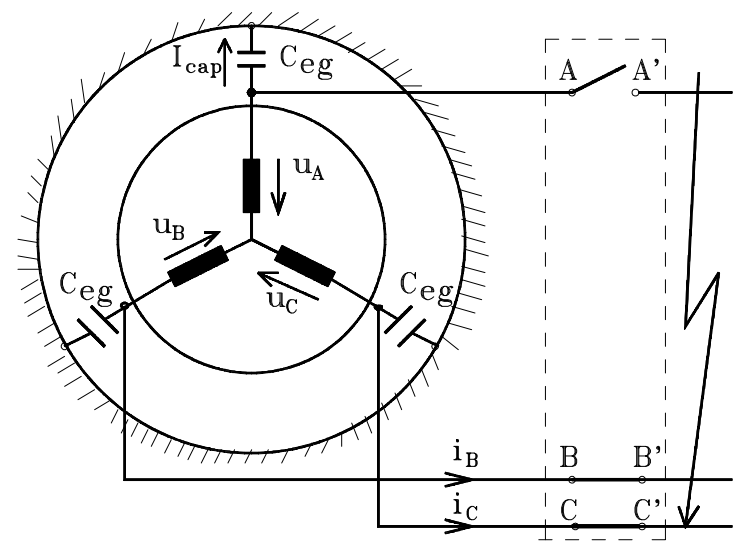

Still, one could note that the capacitance presence will determine further the forming of an oscillatory circuit in which, because of the perturbation, high frequency currents $(10-30 \mathrm{kHz})$ will appear. The magnetic field created in the synchronous generator by these high frequency currents has a spectrum completely different from the useful magnetic fields, rather it having been approached to the leakage field spectrum $[23,26]$. Consequently, this field does not depend on the rotor position or on 
iron saturation or excitation degree, being influenced mainly by the constructive machine elements. In this field, each phase winding will have the same inductance $\mathrm{L}_{\mathrm{g}}$ (see Equation (25)), which is completely different from $\mathrm{L}_{\mathrm{d}}$ " or $\mathrm{L}_{\mathrm{q}}$ ", and the same resistance $\mathrm{R}_{\mathrm{g}}>\mathrm{R}$ (because of the specific phenomena at the oscillation frequency).

If in equation (24) the inductances $L_{d}$ " and $L_{q}$ " are replaced by $L_{g}$, the resistance $R$ is replaced by $R_{g}$, and we take into consideration relation (25), in which:

$$
I_{\text {cap }}=p \cdot C_{e g} \cdot \Delta U_{A}
$$

the following results:

$$
\Delta U_{A}=\left(p \cdot C_{e g}+\frac{1}{R_{g}+p \cdot L_{g}}\right)^{-1} \cdot I_{A}
$$

Since the equivalent phase resistance $R_{g}$ is relatively small (namely, maximum (8-10)R), one could assume that the strong damping of the oscillations of the transient recovery voltage can be justified solely on basis of a conductance of losses of the winding insulation at high frequency. This means we admit a parallel circuit for the capacitor [27-30], as depicted in Figure 6, where:

$$
\begin{gathered}
G_{g}=G_{0}+\omega \cdot C_{(\max )} \cdot \sin \delta_{h} \\
C_{g}=C_{(\max )} \cdot \cos \delta_{h}
\end{gathered}
$$

where $\mathrm{G}_{0}$ denotes the d.c. conductance; $\mathrm{C}_{(\max )}$ the apparent capacitance over the loading cycle (equal to $\mathrm{Q}_{\max } / \mathrm{U}_{\max }$ ); and $\delta_{\mathrm{h}}$ the hysteresis losses' angle (from the cycle area).

Figure 6. Equivalent parallel circuit of the capacitor $\mathrm{C}_{\mathrm{eg}}$

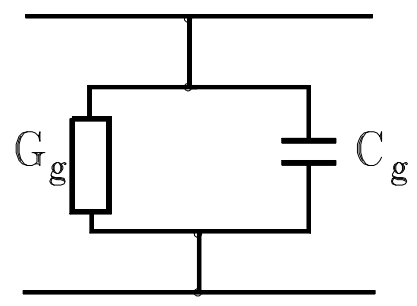

The split of the total losses into conduction losses and hysteresis losses has no relevance in applications. Therefore the equivalent circuits are utilized with elements determined directly through the measurement of losses.

Thus, if $\delta=\pi / 2-\left|\varphi_{e}\right| 1$ is the total losses' angle and $\operatorname{tg} \delta=G_{g} / \omega \cdot C_{g} 2$ is the losses' factor, the following result:

$$
\begin{aligned}
G_{g} & =\omega \cdot C_{g} \cdot \operatorname{tg} \delta \\
G_{g} & =I_{c a p} \cdot \sin \delta / \Delta U_{A} \\
C_{g} & =I_{c a p} \cdot \cos \delta / \Delta U_{A}
\end{aligned}
$$


Without a large error, in applications it may be determined that:

$$
C_{g}=C_{e g} ; \quad G_{g}=\omega \cdot C_{e g} \cdot \operatorname{tg} \delta
$$

Consequently, the operational circuit (27) becomes:

$$
\Delta U_{A}(p)=\left\{G_{g}+p \cdot C_{g}+\frac{1}{R_{g}+p \cdot L_{g}}\right\}^{-1} \cdot I_{A}(p)
$$

With the notation $\Delta \mathrm{I}_{\mathrm{Ab}}=0-\mathrm{I}_{\mathrm{A}}$ for the current variation in the busbar corresponding to phase $\mathrm{A}$ over the current interruption, equation (31) can be rewritten in the form:

$$
\Delta U_{A}=\left\{G_{g}+p \cdot C_{g}+\frac{1}{R_{g}+p \cdot L_{g}}\right\}^{-1} \cdot\left(-\Delta I_{A b}\right)
$$

In Figure 7 we depict the operational circuit corresponding to the equation above. This circuit is actually the phase operational representation of the synchronous generator at the disconnection processes' frequency.

Figure 7. Operational circuit of synchronous generator at first phase current interruption

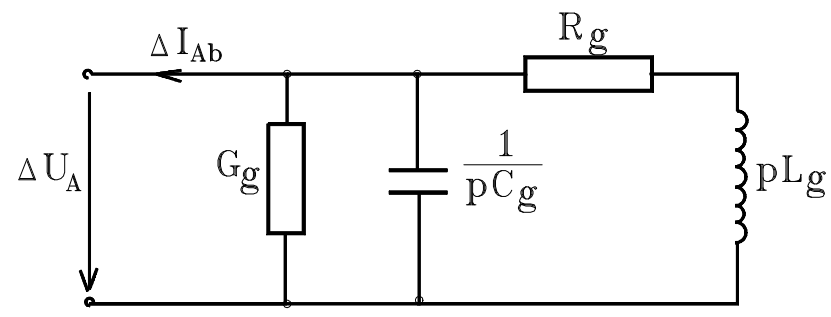

\section{Assessment of Concentrated Equivalent Parameters of Synchronous Generator Windings at Current Interruption Transient Processes}

The method of operational symmetrical components has been applied here to determine the operational equations and configurations of the transient recovery voltage which appears at the generator circuitbreaker terminals after the interruption of a short-circuit fed by the synchronous generator. The TRV which appears after the interruption of a short-circuit fed by the synchronous generator may be considered as an oscillation with the oscillation factor and the rising rate (RR) of the TRV established by the electric parameters of synchronous generator: resistance, inductance and capacitance.

This assessment step is necessary but not sufficient for the modeling of TRV. That is, the structure of the operational impedances of the generator $Z_{g_{+}}(p)$ and $Z_{g_{-}}(p)$ must be assessed taking into consideration the concentrated equivalent parameters of the synchronous generator. This issue is not simple in a sustainable study, since during the interruption current transient processes the electromagnetic phenomena in the generator are complex, and interactions of the generator with the power electric network occur. Modeling the concentrated equivalent parameters of the generator at perturbations caused by current interruption transient processes is imposed. 
Studies on the synchronous generators stator windings during the transient processes caused by current interruptions have led to the hypothesis that they can be seen like long straight conductors [2730]. Thus, the generator capacitance is mainly distributed between each winding and the ferromagnetic core, because both the capacitive coupling among the phase windings and the longitudinal capacitance (among winding turns) are very small and can be neglected. On the other hand, both the mutual inductance among the coils of proximate slots, and the inductance of the frontal terminals zone, can be neglected. Consequently, any phase winding can be seen as a chain of inductances without mutual coupling, having solely capacitances which are uniformly distributed to ground [29-30].

In order to enhance this knowledge, we consider an elementary section (with the length " $d x$ ") from the phase winding of a synchronous generator with the parameters (electric resistance $R^{\prime}$, inductance $L^{\prime}$, electric capacitance $C^{\prime}$ and electric conductance $G^{\prime}$ ) uniformly distributed.

If the conductance $G^{\prime}=0$ (assuming a winding with a perfect insulation) the section " $d x^{\prime \prime}$ corresponds to the representation in Figure 8.

Figure 8. Elementary section of a synchronous generator phase winding

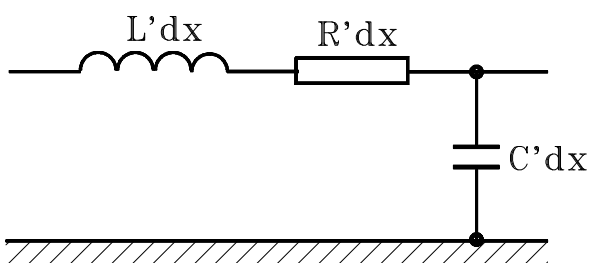

On the entire length $l$, the synchronous generator phase winding (with perfect insulation) is made [29] from identical cells chain of inductances without mutual coupling, having only capacitances which are uniformly distributed to the ground, as in Figure 9.

Figure 9. Long straight conductors corresponding to the generator phase winding

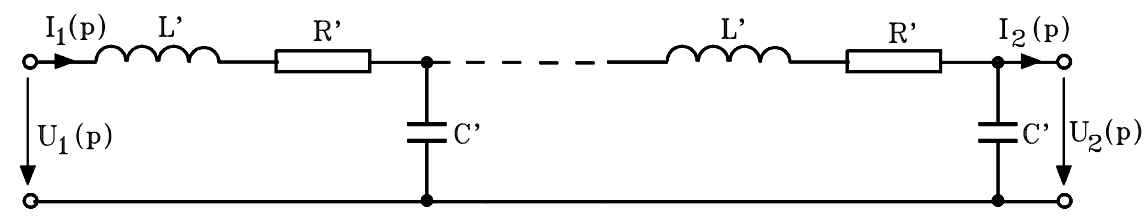

The winding can be seen as a quadrupole, with the operational equation:

$$
\left|\begin{array}{c}
U_{2}(p) \\
I_{2}(p)
\end{array}\right|=\left|\begin{array}{cc}
\operatorname{ch}(\gamma) & Z_{c} \cdot \operatorname{sh}(\gamma) \\
\frac{1}{Z_{c}} \cdot \operatorname{sh}(\gamma) & \operatorname{ch}(\gamma)
\end{array}\right| \cdot\left|\begin{array}{c}
U_{1}(p) \\
I_{1}(p)
\end{array}\right|
$$

where: 


$$
\gamma(p)=\sqrt{\left(R^{\prime}+p \cdot L^{\prime}\right) \cdot\left(0+p \cdot C^{\prime}\right)}=p \cdot \sqrt{L^{\prime} \cdot C^{\prime}} \cdot \sqrt{1+\frac{R^{\prime}}{p \cdot L^{\prime}}}
$$

which is the operational constant of propagation, and

$$
Z_{c}=\sqrt{\frac{R^{\prime}+p \cdot L^{\prime}}{0+p \cdot C^{\prime}}}=\sqrt{\frac{L^{\prime}}{C^{\prime}}} \cdot \sqrt{1+\frac{R^{\prime}}{p \cdot L^{\prime}}}
$$

which represents the wave operational impedance (or the characteristic impedance). By developing in power series the radical and under the restraint of the first two terms, the expressions as before can be approximated by $\gamma(p)=\alpha+p \cdot \beta$, where the damping coefficient is $\alpha=\frac{R^{\prime}}{2} \cdot \sqrt{\frac{C^{\prime}}{L^{\prime}}}$ and the phase constant is $\beta=\sqrt{L^{\prime} \cdot C^{\prime}}$. That is,

$$
Z_{c}(p)=\sqrt{\frac{L^{\prime}}{C^{\prime}}}+\frac{R^{\prime}}{2} \cdot \frac{1}{p \cdot \sqrt{L^{\prime} \cdot C^{\prime}}}
$$

On the other hand, the concentrated equivalent parameters (and, mainly, the longitudinal capacitance $C_{g}$ of a phase winding) are not defined in an absolute mode and they are dependent both on the stator windings connection and on the situation of the neutral point to the ground.

Further, in this sense, we consider the case of star (Y) connection of windings, with grounded neutral $\left(\mathrm{U}_{1}=0\right)$. We determine, as an original contribution, the concentrated equivalent parameters $L_{g}, R_{g}$ and $C_{g}$ of the equivalent configuration represented in Figure 10, based on certain conditions, linked to the analytical methods of conservation of the impedances, of the self frequencies and of the electrostatic energies, respectively.

Figure 10. Equivalent configuration with concentrated parameters

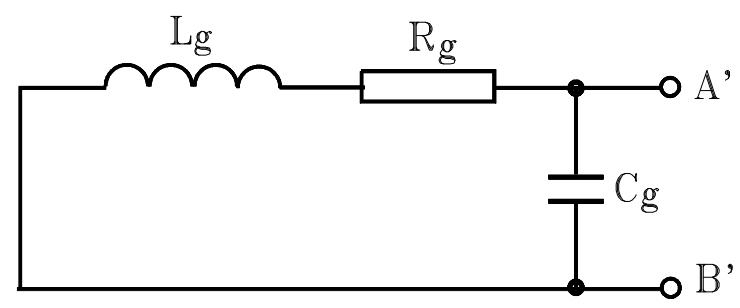

\subsection{Method of Equal Impedances (or Equal Admittances)}

This method is based on the identity of the impedances $Z_{A^{\prime} B^{\prime}}$ (at the terminals of the concentrated parameters equivalent scheme) and $Z_{A B}$ (at the terminals of the line from the Figure 5, having a shortcircuited terminal).

The long lines' equations, equivalent to the synchronous generator phase winding for $U_{l}=0$, are described by: 


$$
\begin{gathered}
U_{2}(p)=Z_{c} \cdot I_{1}(p) \cdot \operatorname{sh} \gamma l \\
I_{2}(p)=I_{1}(p) \cdot \operatorname{ch} \gamma l
\end{gathered}
$$

and, consequently,

$$
Z_{A B}(p)=\frac{U_{2}(p)}{I_{2}(p)}=Z_{c} \cdot t h \gamma l \text { and } \quad Y_{A B}(p)=\frac{c t h \gamma l}{Z_{c}}
$$

With equations (34) and (35), the ratio $\frac{\gamma(p)}{Z_{c}(p)}=p \cdot C^{\prime}$ is achieved, resulting in:

$$
Z_{c}(p)=\frac{\gamma(p)}{p \cdot C^{\prime}}
$$

and:

$$
Z_{A B}(p)=\frac{1}{p \cdot C^{\prime} \cdot l} \cdot \frac{\gamma(p) \cdot l}{c t h \gamma l}=\frac{1}{Y_{A B}(p)}
$$

But, the equivalent admittance at the terminals $A^{\prime} B^{\prime}$ of the representation in Figure 10 is:

$$
Y_{A^{\prime} B^{\prime}}=p \cdot C_{g}+\frac{1}{R_{g}+p \cdot L_{g}}=\frac{p^{2} \cdot L_{g} \cdot C_{g}+p \cdot C_{g} \cdot R_{g}+1}{p \cdot L_{g}+R_{g}}
$$

From the equality of the expressions of the two admittances, $Y_{A^{\prime} B^{\prime}}$ and $Y_{A B}$, we obtain the relation for $C_{g}$ :

$$
C_{g}=\frac{C^{\prime} \cdot p \cdot\left(R_{g}+p \cdot L_{g}\right)-\gamma \cdot \operatorname{th} \gamma l}{p \cdot\left(R_{g}+p \cdot L_{g}\right) \cdot \gamma \cdot t h \gamma l}
$$

Since both the resistance and the inductance interfere with the limitation of the short-circuit currents, their values are adopted as invariant in the two configurations, meaning that $R_{g}=R_{e g}\left(\approx l \cdot R^{\prime}\right)$ and $L_{g}=L_{e g}\left(\approx l \cdot L^{\prime}\right)$. Therefore, equation (42) can be rewritten as:

$$
C_{g}=\frac{C^{\prime} \cdot l \cdot p \cdot\left(R^{\prime}+p \cdot L^{\prime}\right)-\gamma \cdot t h \gamma l}{p \cdot\left(R^{\prime}+p \cdot L^{\prime}\right) \cdot \gamma \cdot l \cdot t h \gamma}
$$

or, taking into account equation (34),

$$
C_{g}=C^{\prime} \cdot l \cdot \frac{\gamma \cdot l-t h \gamma l}{\gamma^{2} \cdot l^{2} \cdot t h \gamma}
$$

Since for $|x|<\pi / 2$ the function thx admits the development [7]: 


$$
\text { thx } x=x-\frac{x^{3}}{3}+\frac{2 x^{5}}{15}-\frac{17 x^{7}}{315}+\ldots
$$

and taking into account solely the first two terms, equation (44) becomes:

$$
C_{g}=C^{\prime} \cdot l^{\prime} \cdot \frac{1 / 3}{l-(1 / 3) \cdot \gamma^{2} \cdot l^{2}}
$$

At the limit, when $\gamma \cdot l \rightarrow 0$, the capacitance $C_{g}$ has the tendency to attain the maximum, and the long lines' equations, equivalent to the synchronous generator phase winding for $U_{l}=0$, are described by:

$$
C_{g}=\frac{C^{\prime} \cdot l}{3}=\frac{C_{e g}}{3}=0.33 \cdot C_{e g}
$$

\subsection{Method of Equal Self-Frequencies}

The method is based on the identity of the self-frequencies corresponding to the two circuits at the resonance [20,23-27].

With the complex variable $p=j \cdot \omega_{0}$, in the absence of the losses $\left(R^{\prime}=0\right)$, the circuits admittances, at the resonance, will be zero. Thus, in the case of the line with distributed parameters, from equation (14) (with $p=j \cdot \omega_{0}$ ), we obtain:

$$
j \cdot \omega_{0} \cdot C^{\prime} \cdot l \cdot \frac{c t h \gamma l}{\gamma \cdot l}=0
$$

or:

$$
\cos j \gamma\left(j \omega_{0}\right) \cdot l=0
$$

Since $\gamma\left(j \omega_{0}\right)=j \cdot \omega_{0} \cdot \sqrt{L^{\prime} \cdot C^{\prime}}$, from equation (49) we obtain:

$$
\omega_{0}=\frac{\pi}{2 \cdot l \cdot \sqrt{L^{\prime} \cdot C^{\prime}}}
$$

The self-pulsation of the concentrated parameters equivalent circuit is:

$$
\omega_{0 g}=\frac{1}{\sqrt{L_{g} \cdot C_{g}}}
$$

From the equality of the two self-pulsations: 


$$
\frac{1}{L_{g} \cdot C_{g}}=\frac{\pi^{2}}{4 \cdot L^{\prime} \cdot l \cdot C^{\prime} \cdot l}
$$

Under the assumption $L_{g}=L^{\prime} \cdot l$, we obtain the equivalent capacitance value:

$$
C_{g}=\frac{4}{\pi^{2}} \cdot\left(C^{\prime} \cdot l\right)=0.406 \cdot C_{e g}
$$

where $C_{e g}=C^{\prime} \cdot l$ it is the total capacitance distributed along the synchronous generator phase winding.

\subsection{Method of Equal Electrostatic Energies}

The starting point of the method is based on the statement that two equivalent circuits, for the same voltage amplitude, equally store electrostatic energies [20,23-27]. Thus, admitting a linear variation of the voltage along the winding (see Figure 11), with:

$$
U_{2}-U_{1}=\Delta U
$$

Into an elementary capacitance $C^{\prime}=C / l$, situated at the distance $x$ from the winding terminal, the stored electrostatic energy is $W_{x}=\frac{1}{2} \cdot C^{\prime} \cdot U_{x}^{2}$, where:

$$
U_{x}=U_{l}+\frac{x}{l} \cdot \Delta U
$$

Figure 11. Voltage variation along a phase winding

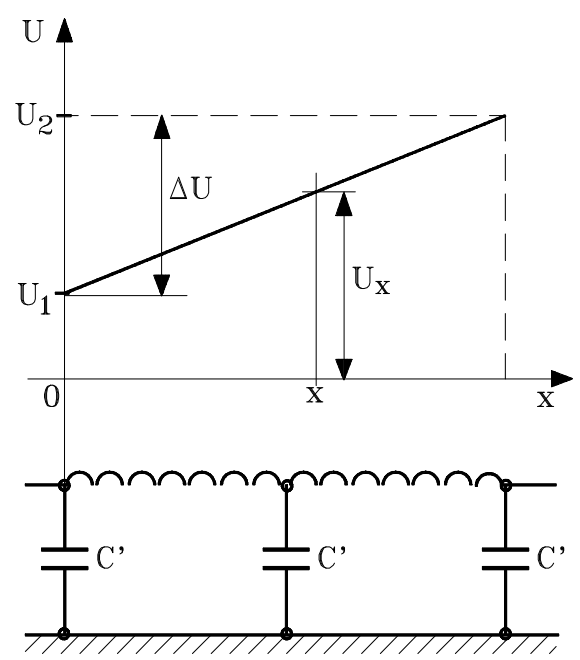

The total energy stored in the entire winding capacitance is determined with: 


$$
W=\int_{0}^{l} W_{x} \cdot d x=\frac{1}{2} \cdot C^{\prime} \cdot l \cdot\left[U_{1}^{2}+{ }_{U_{1}} \cdot \Delta U+\frac{1}{3}(\Delta U)^{2}\right]
$$

Since $U_{1}=U_{2}-\Delta U$, equation (56) becomes:

$$
W=\frac{1}{2} \cdot U_{2}^{2} \cdot\left(C^{\prime} \cdot l\right) \cdot\left[1-\frac{\Delta U}{U_{2}}+\frac{1}{3} \cdot\left(\frac{\Delta U}{U_{2}}\right)^{2}\right]
$$

It is emphasized that the electrostatic energy stored in the concentrated capacitance $C_{g}$ at the voltage $U_{2}$ is $\frac{1}{2} \cdot U_{2}^{2} \cdot C_{g}$, and by identification with equation (57) we obtain:

$$
C_{g}=C^{\prime} \cdot l \cdot\left[1-\frac{\Delta U}{U_{2}}+\frac{1}{3} \cdot\left(\frac{\Delta U}{U_{2}}\right)^{2}\right]
$$

In the case of the grounded windings, $U_{1}=0$ and $U_{2}=\Delta U$. Consequently, from the expression (58) we find again that $C_{g}=\frac{1}{3} \cdot\left(C^{\prime} \cdot l\right)$.

\section{Conclusions}

This paper extends other studies of the authors which have examined the exergetic transformation chain at the interruption current transient process in an electric power system that comprises the generator circuit-breaker.

The method of operational symmetrical components has been applied to assess the operational equations and configurations of the transient recovery voltage which appears at the generator circuitbreaker terminals after the interruption of a three-phase short-circuit fed by a synchronous generator.

Applying the method of operational symmetrical components to determine the TRV which appears after the interruption of a short-circuit fed by the synchronous generator represents an original contribution of the authors of this study. Modeling transient recovery voltage of circuits emphasizes aspects with direct implication on commutation equipment. Thus, the o.s.c. method can be applied at the poles of any breaker, for any eliminated fault type, if the network configuration and elements are known.

Further, these findings have been followed by the assessment of the structure of the operational impedances of the generator $Z_{g^{+}}(p)$ and $Z_{g_{-}}(p)$ taking into consideration the concentrated electric parameters of the synchronous generator. During the interruption current transient processes, the electromagnetic phenomena in the synchronous generator are complex, and interactions of the generator with the electric network occur. Modeling of concentrated equivalent parameters of the synchronous generator at perturbations caused by current interruption transient processes is achieved in this study through an approach based on the model of an industrial ecosystem. Thus, adopting the pattern of ideal synchronous machine and applying the overlapping principle and the coordinate transformation, we obtained the operational equations of synchronous generator at sudden load variations.

As another main conclusion the importance is highlighted of the components $(\alpha, \beta)$. They provide a useful physical point of view concerning the phase current interruption, highlighting the dualist view 
of industrial ecology, according to which the behavioral analysis of an industrial ecosystem can be performed in two distinct reference frames.

These findings represent a necessary but not sufficient step for TRV modeling. Further modeling the concentrated equivalent parameters of the generator at perturbations caused by current interruption transient processes is achieved in this study through an approach based on sustainability concepts.

Thus, using the equal impedance and equal electrostatic energy methods we obtain for the synchronous generator phase winding equivalent capacitance $C_{g}$ the value $0.330 \cdot C_{e g}$. Applying the equal self-frequency method results in the value $0.406 \cdot C_{e g}$ for $C_{g}$. We conclude that the value of the equivalent capacitance $C_{g}$ of a synchronous generator phase winding (depending on the applied method) varies in the range of $0.330 \cdot C_{e g}$ to $0.406 \cdot C_{e g}$, and these findings should be taken into consideration for modeling the TRV on the basis of operational impedances of the synchronous generator during the interruption processes transient processes.

\section{Conflict of Interest}

The authors declare no conflict of interest.

\section{References and Notes}

1. Dincer I.; Rosen M.A. Exergy: Energy, Environment and Sustainable Development, $2 \mathrm{~d}$ ed. Oxford, UK: Elsevier, 2013.

2. Rosen, M.A. A Concise Review of Energy-Based Economic Methods, Proc. $3^{\text {rd }}$ IASME/WSEAS Int. Conf. on Energy \& Environment, Cambridge, UK, February 23-25, 2008, pp.136-142.

3. Wall, G. Exergy tools, Proc. Inst. Mechanical Engineers, Part A: J. Power and Energy, Vol. 217, 2003, pp.125-136.

4. Bulucea, C.A.; Nicola, D.A.; Mastorakis, N.E.; Rosen, M.A. Understanding Electric Industrial Ecosystems through Exergy, Recent Researches in Energy and Environment: Proc. 6th IASME/WSEAS Int. Conf. on Energy and Environment, Cambridge, UK, February 20-25, 2011, pp. 182-191.

5. Nicola, D.A.; Bulucea C.A. Concerning the Determination of the Duration of the Unilateral Current to the Sudden Short-circuit to the Synchronous Generators; Part I: Analytical Estimation. Proc. of the International Conference of Brasov: OPTIM '94, Brasov, Romania, May 1994, pp. 237-242.

6. Nicola, D.A.; Bulucea C.A. Concerning the Determination of the Duration of the Unilateral Current to the Sudden Short-circuit to the Synchronous Generators; Part II: Geometrical Representation in Powers Plane. Proc. of the International Conference of Brasov: OPTIM '94, Brasov, Romania, May 1994, pp. 243-246.

7. Rosen, M.A.; Dincer, I. Exergy analysis of waste emissions, Int. J Energy Research, Vol. 23, 1999, pp. 1153-1163.

8. Smeets, R.P.P; Barts, H.D.; Zehnder, L. Extreme stresses on generator circuit breakers. A3-304 IGRE 2006. 
9. Ježek, V. Perspective generator circuit breaker. Intensive Programme "Renewable Energy Sources”, Železná Ruda-Špičák, University of West Bohemia, Czech Republic, May 2010. Available

online:

http://home.zcu.cz/ tesarova/IP/Proceedings/Proc_2010/Files/021\%20IP2010\%20Jezek.pdf (accessed on March 2012).

10. Ježek, V. Short-circuit capability of generator circuit breaker. Intensive Programme "Renewable Energy Sources", Železná Ruda-Špičák, University of West Bohemia, Czech Republic, May 2011. Available online: http://home.zcu.cz/ tesarova/IP/Proceedings/Proc 2011/Files/Jezek.pdf (accessed on June 2012).

11. Leung, S.Y.; Snider, L.A.; Wong, C.S.M. SF6 Generator Circuit Breaker Modeling. Paper Presented at the International Conference on Power Systems Transients (IPST'05), Montreal, Canada, June 19-23, 2005, Paper No. IPST05-243. Available online: http://www.ipst.org/TechPapers/2005/IPST05 Paper243.pdf (accessed on March 2012).

12. Eidinger, A. Interruption of high asymmetric short-circuit current having delayed zeros - an acute problem for generator breaker, IEEE Trans. PAS 91, 1972 (4).

13. Dufournet, D.; Willieme, J.M.; Montillet, G.F. Design and implementation of an SF6 interrupting chamber applied to low range generator circuit breakers suitable for interruption of current having a non-zero passage. IEEE Transactions on Power Delivery, Volume 17, No. 4, October 2002, pp. 963-968.

14. Rodstein, I. Electrical Control Equipment, English translation, Mir Publishers, 1974.

15. Dufournet, D.; Montillet, G.F. Transient recovery voltages requirements for system source fault interrupting by small generator circuit breakers. IEEE Transactions on Power Delivery, Vol. 17, No. 2, April 2002.

16. Bulucea, C.A.; Rosen, M.A.; Nicola, D.A.; Mastorakis, N.E.; Bulucea, C.A., Analyzing the Interruption Processes in the Generator Circuit Breaker through Sustainability Concepts, In Proceedings of the 2nd World Sustain. Forum, 1-30 November 2012; Sciforum Electronic Conferences Series, 2012, Section D: Energy Efficiency and Renewable Energy Sources, Published online: 29 October 2012

17. Bulucea C.A., Rosen M.A., Nicola D.A., Mastorakis N.E. and Bulucea C.A., Approaching the Processes in the Generator Circuit Breaker at Disconnection through Sustainability Concepts, Sustainability 2013, 5, 1161-1176, doi:10.3390/su5031161

18. Bulucea C.A., Rosen M.A., Nicola D.A., Mastorakis N.E. and Bulucea C.A., Addressing Some Sustainability Aspects of Electric Power Systems Comprising Generator Circuit-Breakers, In Recent Advances in Energy and Environmental Management, Proceedings of $8^{\text {th }}$ WSEAS International Conference on Energy and Environment (EE'13), Rhodes Island, Greece, July 16-19, 2013, pp. 43-50.

19. Bulucea C.A., Rosen M.A., Nicola D.A., Mastorakis N.E. and Bulucea C.A., On the Influence of Synchronous Generator on Disconnection Processes in Electric Power Systems Comprising Generator Circuit Breakers, In Recent Researches in Electric Power and Energy Systems, Proceedings of $13^{\text {th }}$ WSEAS International Conference on Electric Power Systems, High Voltages, Electric Machines (POWER'13), Chania, Crete Island, Greece, August 27-29, 2013, pp. 50-58. 
20. Coulon, I.; Jufer, M. Introduction à l'électrotechnique (Introduction to Electrotechnics), 5th ed., Piesses Politechniques Romandes, Lausanne, 1989.

21. Braun, A.; Huber, H.; Suiter, H., Determination of the transient recovery voltage occurring across generator circuit breaker in large modern power station, International Conference on Large High Voltage Electric System, 25.08-2.09 1976, 13-03.

22. Sulkowski, J., Analysis of stresses on switching device located between the generator and the main transformer of a nuclear plant, IEEE Trans. Pas, A 79 532, 1979.

23. Nicola, D.A.; Bulucea, C.A. Electrotehnica, masini si echipamente electrice (Electrotechnics, Electrical Machines and Equipment), SITECH Publishing House, Craiova, Romania, 2005.

24. Bulucea, C.A.; Nicola, D.A. Introducere în electrotehnica si echipamente electrice (Introduction to Electrotechnics and Electrical Equipment), SITECH Publishing House, Craiova, Romania, 2004.

25. Bala, C., Masini electrice (Electric Machines), EDP Publishing House, Bucharest, 1979.

26. Boldea, I.; Atanasiu, G., Analiza unitara a masinilor electrice (Unitary Analysis of Electrical Machines), Ed. Academiei Publishing House, Bucharest, 1983.

27. Clarke, E., Analiza circuitelor sistemelor electroenergetice (Circuit Analysis of A-C Power Systems), Translation from English, Ed. Tehnica Publishing House, Bucharest, 1974.

28. Park, R.H., Two reaction theory of synchronous machines; generalized method of analysis, AIEE Trans., 1929, 48, (I), pp. 716-728.

29. Iacobescu, G., Alegerea parametrilor L si C ai lantului de cuadripoli circuit echivalent al infasurarii unui alternator pentru calculul procesului de restabilire a tensiunii la bornele intreruptorului (Choice of parameters $\mathrm{L}$ and $\mathrm{C}$ of equivalent quadrupole circuit of a generator winding for transient recovery voltage process at a circuit-breaker terminals), IPB Scientific Bulletin, Tome XXVIII, No. 6, 1966.

30. Iordanescu, I; Tudose, M.; Iacobescu, G., Retele si sisteme electrice (Electric Networks and Systems), EDP Publishing House, Bucharest, 1979.

(C) 2013 by the authors; licensee MDPI, Basel, Switzerland. This article is an open access article distributed under the terms and conditions of the Creative Commons Attribution license (http://creativecommons.org/licenses/by/3.0/). 